TITLE:

\title{
Effects of pulse duration upon the plume formation by the laser ablation of $\mathrm{Cu}$ in water
}

$\operatorname{AUTHOR}(\mathrm{S}):$

Oguchi, H; Sakka, T; Ogata, YH

\section{CITATION:}

Oguchi, $\mathrm{H}$...[et al]. Effects of pulse duration upon the plume formation by the laser ablation of Cu in water. JOURNAL OF APPLIED PHYSICS 2007, 102(2): 023306.

ISSUE DATE:

2007-07-15

URL:

http://hdl.handle.net/2433/50405

\section{RIGHT:}

Copyright 2007 American Institute of Physics. This article may be downloaded for personal use only. Any other use requires prior permission of the author and the American Institute of Physics. 


\title{
Effects of pulse duration upon the plume formation by the laser ablation of $\mathrm{Cu}$ in water
}

\author{
Hisayuki Oguchi, Tetsuo Sakka, ${ }^{\text {a) }}$ and Yukio H. Ogata \\ Institute of Advanced Energy, Kyoto University, Uji, Kyoto 611-0011, Japan
}

(Received 7 March 2007; accepted 8 June 2007; published online 25 July 2007)

\begin{abstract}
Time-resolved images of an ablation plume obtained at different pulse durations have been measured for the liquid-phase laser ablation of a $\mathrm{Cu}$ plate, and the emission intensity and size of the plume have been discussed. In some plume images, high-density bright spots were observed. The size of the light-emitting region increases and the bright spots disappear for the irradiation with a 150-ns pulse. We conclude that consecutive excitation by the later part of a longer pulse expands the plume and reduces plume density. These results are consistent with our previous result that less-broadened atomic line spectra with high emission intensity were obtained with a 150-ns-pulse irradiation. () 2007 American Institute of Physics. [DOI: 10.1063/1.2759182]
\end{abstract}

\section{INTRODUCTION}

The emission spectra of a laser ablation plume provide information on the elements present in a sample target. These spectra can be used for surface elemental analysis and the method can be classified as a variation of the analytical method generally called laser-induced breakdown spectroscopy. This method has the advantage of quick monitoring with high spatial resolution and lack of complex sample pretreatment. $^{1-5}$

Our goal is to apply this analytical method to the in situ monitoring of various surfaces being processed in liquid, such as electrode surfaces under electrodeposition. However, the quality of the earlier spectra when the target is placed in liquid is too poor for practical spectrochemical analysis, i.e., the spectral profile is broadened and superposed by the continuous spectrum. ${ }^{6-11}$ To improve the quality of the spectra, researchers have subjected a solid target in water to doublepulse irradiation, which gives narrow and clear spectral lines compared with single-pulse irradiation. ${ }^{4,5}$

Previously, we succeeded in acquiring narrow spectral lines by single-pulse irradiation at a pulse duration of 40-150 ns. ${ }^{12-14}$ Figure 1, cited from our previous work, ${ }^{13}$ shows emission spectra obtained for a $\mathrm{Cu}$ plate immersed in pure water with 150 - and 20-ns pulse irradiation for various delay times. In the case of a 20-ns pulse, the two emission lines at around $325 \mathrm{~nm}$ broaden and merge irrespective of delay time. On the other hand, a 150-ns pulse gives a narrow emission line profile at a delay time greater than approximately $500 \mathrm{~ns}$, although the two emission lines broaden and merge at a delay time shorter than $400 \mathrm{~ns}$, e.g., $300 \mathrm{~ns}$. Although a short pulse leads to efficient ablation, a pulse as short as 20 ns gives very weak emission, as well as broad lines superposed on a continuous spectral profile. The result showing that a long pulse induces intense emission, while showing inefficient ablation, is seemingly contradictory.

The mechanism explaining why a long pulse works in obtaining narrow spectral lines has not been investigated so

\footnotetext{
${ }^{a)}$ Author to whom correspondence should be addressed; electronic mail: t-sakka@iae.kyoto-u.ac.jp
}

far. We believe that the time evolution of a plume during and immediately after the laser irradiation should give important information on the mechanism ${ }^{15}$ and that the effect of pulse duration should be compared.

In this work, time-resolved images of an ablation plume have been taken using a high-speed camera based on an intensified charge coupled device (ICCD), and the plume size and spatial distribution, as well as the emission intensity, have been discussed to verify the effect of pulse duration.

\section{EXPERIMENT}

The $\mathrm{Cu}$ plate $(99.96 \%$, Nilaco Inc., Ltd.) used as an ablation target was placed horizontally in a glass cell filled with pure water. The depth from the water surface to the target surface was approximately $10 \mathrm{~mm}$. A home-built multimode Nd:yttrium-aluminum-garnet (YAG) laser oscillator operated in the $Q$-switch pulse mode at $1064 \mathrm{~nm}$ at a repetition rate of $\sim 1 \mathrm{~Hz}$ was used as an excitation pulse source. Pulse duration, defined by the full width at half maximum (FWHM),
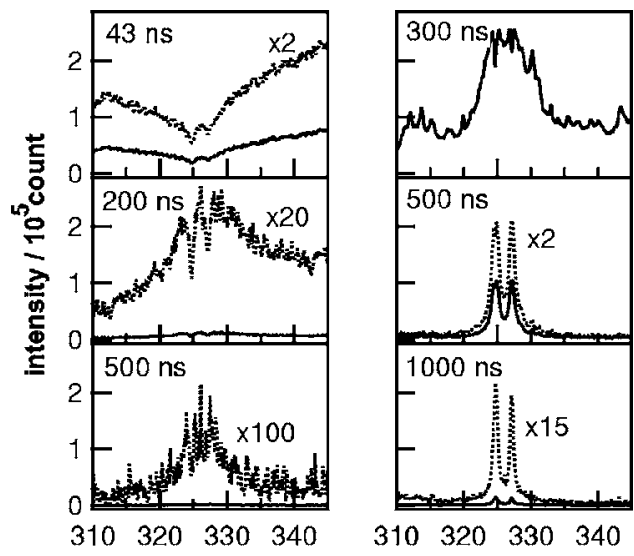

(a) wavelength $/ \mathrm{nm} \quad$ (b)

FIG. 1. Emission spectra obtained in water for $\mathrm{Cu} \mathrm{I}{ }^{2} \mathrm{~S}_{1 / 2}-{ }^{2} \mathrm{P}_{3 / 2}^{o}$ (or ${ }^{2} \mathrm{P}_{1 / 2}^{o}$ ) fine-structure doublet at 324.75 and $327.40 \mathrm{~nm}$ for various delay times. The gate width of data acquisition was $5000 \mathrm{~ns}$. The energy of the ablation pulse was adjusted to $1.7 \mathrm{~mJ}$. Each spectrum is an accumulation of the spectra obtained by five consecutive pulse shots. Pulse duration was (a) 20 and (b) 150 ns. 
was controlled to $20,50,90$, or $150 \mathrm{~ns}$ by adjusting the voltage applied to the flash lamp for lasing. ${ }^{12}$ The operation near the lasing threshold gives a longer pulse than that at a higher population inversion ratio. ${ }^{16}$ For the longer pulse to have a pulse energy adequate for forming an ablation plume, the beam from the oscillator was further amplified using a $\mathrm{Nd}$ :YAG light amplifier. The beam diameter of the laser pulse immediately after the amplifier was approximately 4 $\mathrm{mm}$. The final pulse energy was adjusted to $16.3 \mathrm{~mJ}$, that is, the peak power of a shorter pulse was higher and vice versa. The laser pulse was focused by a $60-\mathrm{mm}$-focal-length lens onto a target immersed in pure water. The irradiation spot was not scanned and, hence, we did not have fresh surface for each pulse. However, the target was moved manually when the emission intensity was lowered by the drilling effect of the ablation. To avoid absorption of the laser pulse energy by particulates formed by former shots, the water was replaced frequently. Sideview images of the ablation plume were taken using a camera based on a gated ICCD (PI-MAX, Princeton Instruments) with a $10 \times$ objective lens (UMPLFl10XBD, Olympus). The trigger of the ICCD was obtained by detecting part of the laser beam right after the laser amplifier using a $p-i-n$ silicon photodiode. The laser pulse was given an approximately 39-m light path before the irradiation, which corresponded to an approximately 130-ns delay from the ICCD triggering. As a result, we were able to take an image before the arrival of the pulse at the target surface. When the emitting light saturates the ICCD, a 95\%-cut neutral density (ND) filter was placed between the target and the objective lens. The duration of the gate was set to $5 \mathrm{~ns}$ and the time delay from the laser oscillation, which was $130 \mathrm{~ns}$ before the irradiation of the target, was varied from 100 to $2000 \mathrm{~ns}$. The resolution of the image obtained by this system was $11.8 \mu \mathrm{m}$, which was checked using a resolution test target (Edmund Scientific, CJ43488).

\section{RESULTS AND DISCUSSION}

Figures 2 and 3 show images of the plume obtained by irradiating a target immersed in pure water with a laser with pulse durations of 20 and $150 \mathrm{~ns}$. The delay time from the laser oscillation was noted in each image. Note that the trigger of the ICCD was obtained at the instant of laser oscillation and that the laser pulse passed through a 39-m light path, which means that the laser pulse arrived at the target surface 130 ns later than the trigger of the ICCD. That is, a 130-ns delay means that an image is taken right at the start of the irradiation with a pulse. The intensity scale of each image is optimized to give the maximum clarity and a scale bar is shown in each image. The white broken line shows the approximate position of the target surface. For the irradiation with the 20-ns pulse, the image obtained at a delay time of $180 \mathrm{~ns}$ is so intense that we used a 95\%-cut ND filter. Similarly, the filter was used for the images taken at delay times of 200, 300, and $400 \mathrm{~ns}$ for the irradiation with the 150-ns pulse.

No emission from the ablation plume was detected at a delay time of $100 \mathrm{~ns}$, which is before the arrival of the pulse at the target surface. By the irradiation with a 20 -ns pulse the
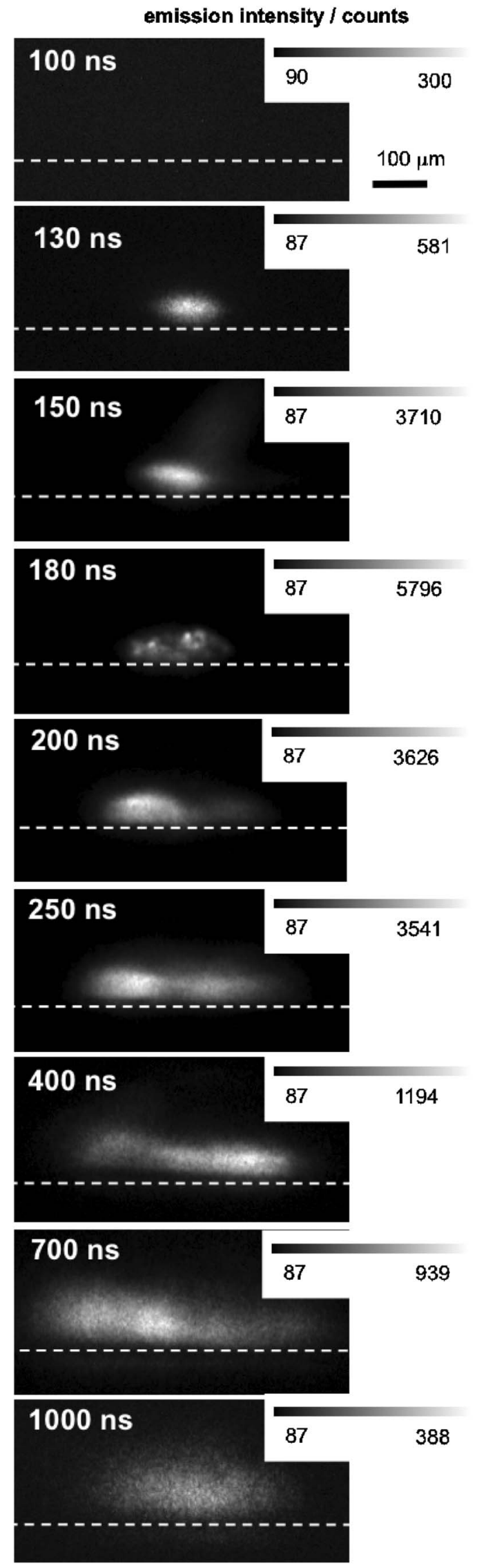

FIG. 2. Images of ablation plumes obtained by the 20 -ns pulse. The ICCD gate delay from the laser oscillation is noted in each image. The gradation scale of each image is optimized for the highest clarity. For the delay time of $180 \mathrm{~ns}, 95 \%$ of the light was cut by a ND filter to avoid saturation of the detector. The white broken line indicates the target surface. 
emission intensity / counts
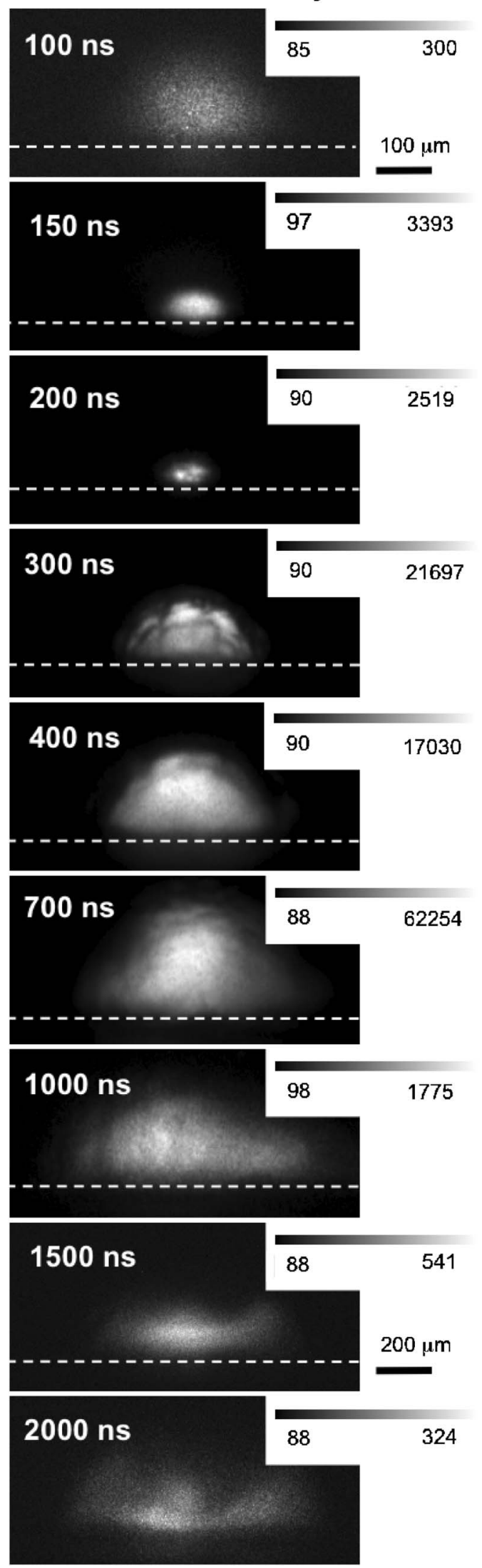

FIG. 3. Images of ablation plumes obtained by the 150-ns pulse. The ICCD gate delay from the laser oscillation is noted in each image. The gradation scale of each image is optimized for the highest clarity. For the delay times of 200,300 , and $400 \mathrm{~ns}, 95 \%$ of the light was cut by a ND filter to avoid saturation of the detector. The white broken line indicates the target surface. The size of the images for 1500 and 2000 ns are reduced into half. emission appeared at $130 \mathrm{~ns}$ and emission intensity increased for a short period. The most intense emission was observed at a delay time of $180 \mathrm{~ns}$. In most of the images obtained by the irradiation with the $20 \mathrm{~ns}$ pulse, several spots with bright centers are clearly seen. It is shown in Fig. 1 that the emission spectra obtained by the irradiation with the 20-ns pulse are broad and continuous. One of the reasons for this may be the white light emission from the high-density bright spots. The ablation plume expands with increasing time and lasts up to $1000 \mathrm{~ns}$. In the case of the 20-ns-pulse irradiation, the bright spots appear irrespective of delay time, which is consistent with the emission of broadened spectra observed for the entire delay time.

In the case of the irradiation with the 150-ns pulse, a weak light is observed at the center of the image at a delay time of $100 \mathrm{~ns}$ (Fig. 3). This is not attributed to the emission of the ablation plume, but to the stray light originating from the flash lamp in the laser amplifier. The emission from the ablation plume appears at a delay time of $150 \mathrm{~ns}$ and then its intensity increases gradually with increasing time. The highest intensity was obtained at $400 \mathrm{~ns}$ and the emission lasted up to $2000 \mathrm{~ns}$. In the images obtained at delay times of 200 and $300 \mathrm{~ns}$, bright spots were observed, as has been observed also for the irradiation with the 20-ns pulse. This suggests that the ablation plume produced by the $150-\mathrm{ns}$ pulse also contains high-density spots, at least in the early stages. At delay times of $400 \mathrm{~ns}$ and later, however, no such bright spots are observed and the emission is rather homogeneously distributed with the brightest region at the center of the ablation plume. Also, the emission region looks hemispherical. This notable difference in the appearance of the bright spots in the plume images suggests the further excitation effect of the plume by the 150-ns pulse, which is less important in the case of the 20-ns-pulse irradiation. The earlier part of the $150-n s$ pulse induces laser ablation, resulting in the formation of an ablation plume that contains high-density spots. The later part of the pulse, in the case of the 150-ns pulse duration, continuously excites the plume probably through inverse bremsstrahlung, leading to the fractionation of highdensity spots into a comparatively lower-density gaseous state, while keeping the atoms and ions excited. At a delay time of $400 \mathrm{~ns}$ or later, therefore, the atoms in the plume still emit light efficiently, and show an emission with a smoothly distributed intensity profile. This interpretation is supported by the results of emission spectra. By the irradiation with a 150 -ns pulse, the emission spectra obtained before the delay time of 300 ns shows a broad and continuous profile, whereas after $500 \mathrm{~ns}$ they show sharp line profiles (Fig. 1). The timing of the transition from the highly broadened spectra to the narrow and sharp spectral lines corresponds to the timing of the disappearance of the bright spots in the plume images. These results are consistently explained by the direct excitation of the plume by the later part of the pulse, if the pulse duration is long enough, such as $150 \mathrm{~ns}$.

The bright spots observed in the plume image may have originated from the particulates emitted directly from the surface. Since the plume temperature is as high as $5000 \mathrm{~K},{ }^{10,11}$ the particulates must not be in a stable solid state. However, 

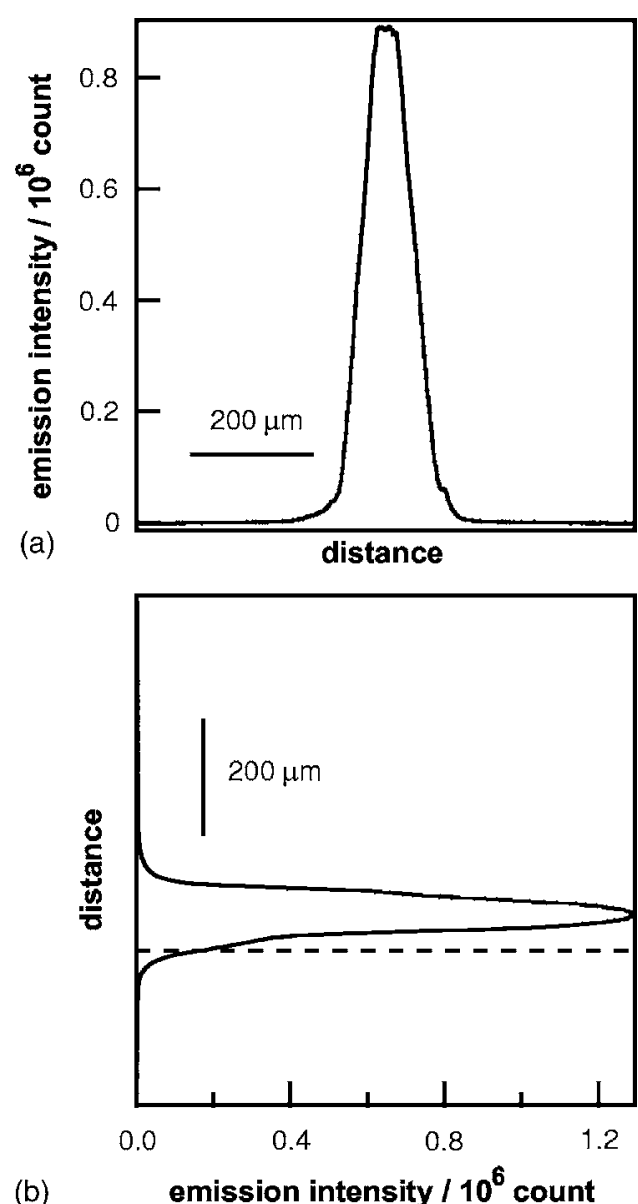

FIG. 4. Examples of intensity distributions (a) in the lateral direction and (b) in the vertical direction. They are obtained by the integration of emission images (a) in the direction normal to the target surface and (b) in the direction parallel to the target surface, respectively. The irradiation pulse duration was $150 \mathrm{~ns}$ and the delay time from the laser oscillation was $400 \mathrm{~ns}$ for these examples. The broken line indicates the target surface.

the particulates emitted directly from the surface may form high-density spots which give an intense blackbody radiation.

Figure 4 shows the results of the integration of a twodimensional intensity image into a one-dimensional intensity distribution. In Fig. 4(a), the integration was performed in the direction normal to the surface, whereas in Fig. 4(b), the results obtained by integration in the direction parallel to the target surface are given. We obtain the size of the emitting region or of the plume by this method. Further integration of the intensity distributions shown in Fig. 4 gives the total emission intensity of the whole plume. The integrated emission intensities obtained at various pulse durations, i.e., 20, 50, 90, and $150 \mathrm{ns,} \mathrm{are} \mathrm{compared.} \mathrm{In} \mathrm{Fig.} \mathrm{5,} \mathrm{the} \mathrm{total} \mathrm{inten-}$ sity, as well as the ablation pulse intensity, is plotted as a function of delay time. The total intensity has a maximum, or a peak, always after the ablation pulse. The peak height of the total emission intensity increases with increasing pulse duration. Irrespective of pulse duration, integrated emission intensity increases during the irradiation of the laser pulse. After the complete extinction of the pulse, the intensity starts to decrease for all the pulse durations examined. However, the rate of the decrease is significantly slow for a long pulse

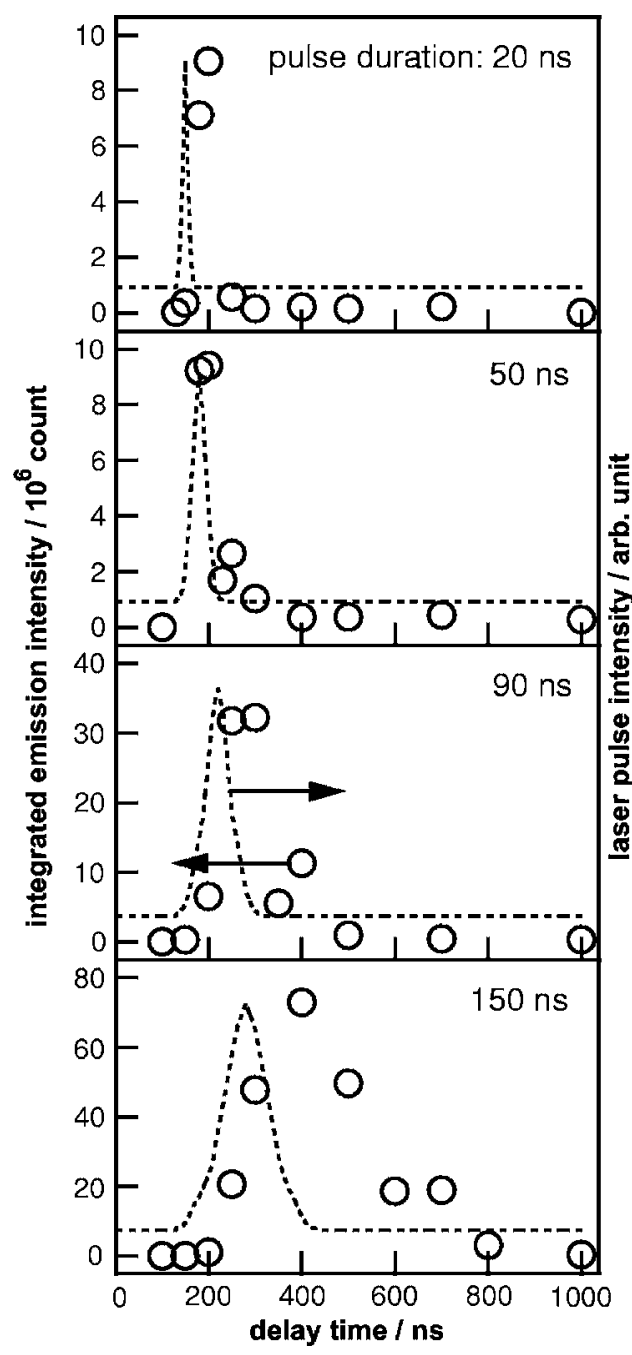

FIG. 5. Integrated total emission intensities obtained by the irradiation with the 20-, 50-, 90-, and 150-ns pulses. Open circles show integrated emission intensities plotted as a function of delay time. Broken lines represent the intensity of the laser pulses.

irradiation. The long lifetime and high intensity of the emission from the ablation plume are considered to be due to the direct excitation of the plume by the later part of the long pulse. Previously, ${ }^{12}$ we reported that the emission intensity of the spectral lines of $\mathrm{Cu}$ atoms increases with pulse duration, which agrees with the present results.

The size of the ablation plume was determined from the FWHM of the distribution obtained by integrating a plume image in a certain direction. In Fig. 6, the FWHM of the distribution in the lateral direction obtained by such integration in the direction normal to the target surface is shown as well as the intensity profile of the irradiation laser pulse. The plume tends to expand for a while even after the extinction of the irradiation of laser pulse, although the emission intensity decreases rapidly, as shown in Fig. 5. That is, the peak height of the spatial intensity distribution obtained by the one-dimensional integration of the image decreases in this time range, whereas the width of the distribution increases. This means that the plume is expanding while being extinguished. In addition, the rate of the expansion in the lateral direction observed after the extinction of the irradiation pulse 


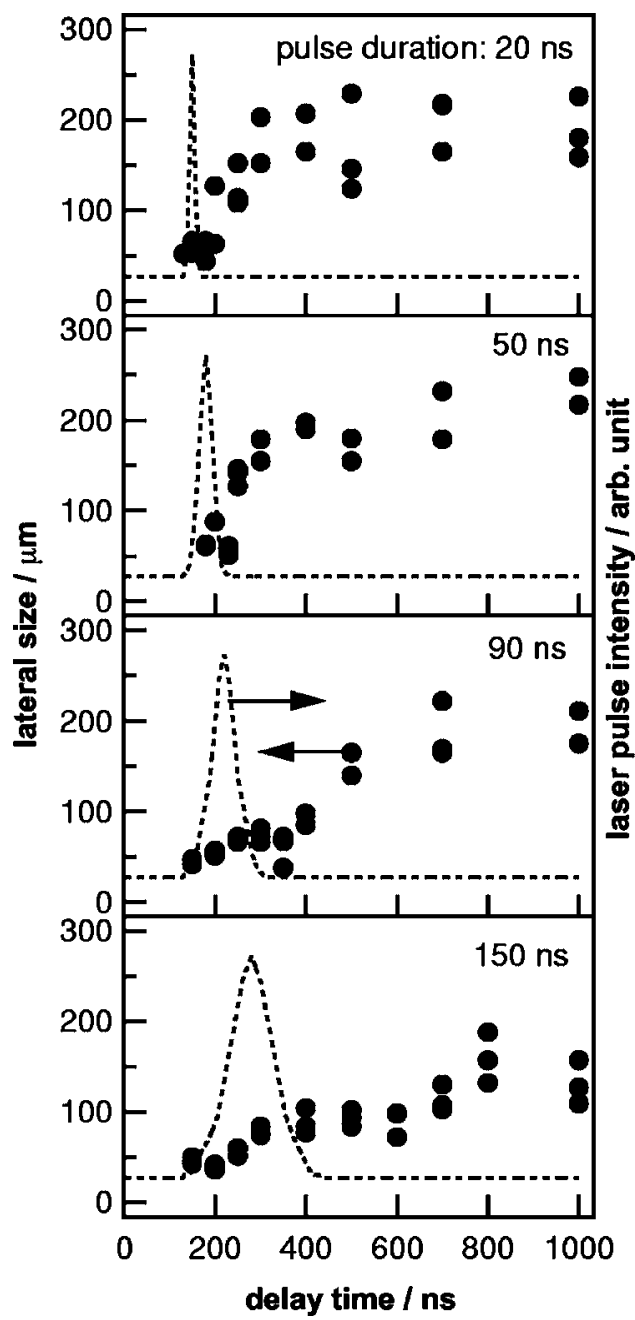

FIG. 6. The lateral size of the ablation plumes obtained by the irradiation of the 20-, 50-, 90-, and 150-ns pulses. Filled circles show the lateral size as a function of delay time, while the broken line represents the intensity profile of a laser pulse.

is higher for a shorter pulse duration. Since a short pulse is expected to give extremely dense and high pressure nascent plume, the results suggest that the fast expansion within a short time favors expansion in the lateral direction. Because we are looking at lateral emission, the optical thickness through the optical pathway along the measurement line is still very high, and therefore the emission intensity suffers from a strong self-absorption, being not as intense as expected from the high density of the plume for a 20 -ns pulse.

In Fig. 7, the size of the ablation plume in the direction normal to the target surface is plotted as a function of time for various irradiation pulse durations. For all the pulse durations, the size increases steeply during the pulse irradiation; however, the increase is very slow after the extinction of the irradiation pulse. For the 150-ns pulse used as an ablation laser, plume size continuously increases for a long time, and the final plume size becomes as large as $60 \mu \mathrm{m}$. As mentioned earlier, this is explained by the consecutive excitation of the plume by the later part of a long nanosecond pulse, causing further excitation of the plume to give considerable amount of excited atoms produced by the homogenization of the plume as well as the direct excitation of ablated

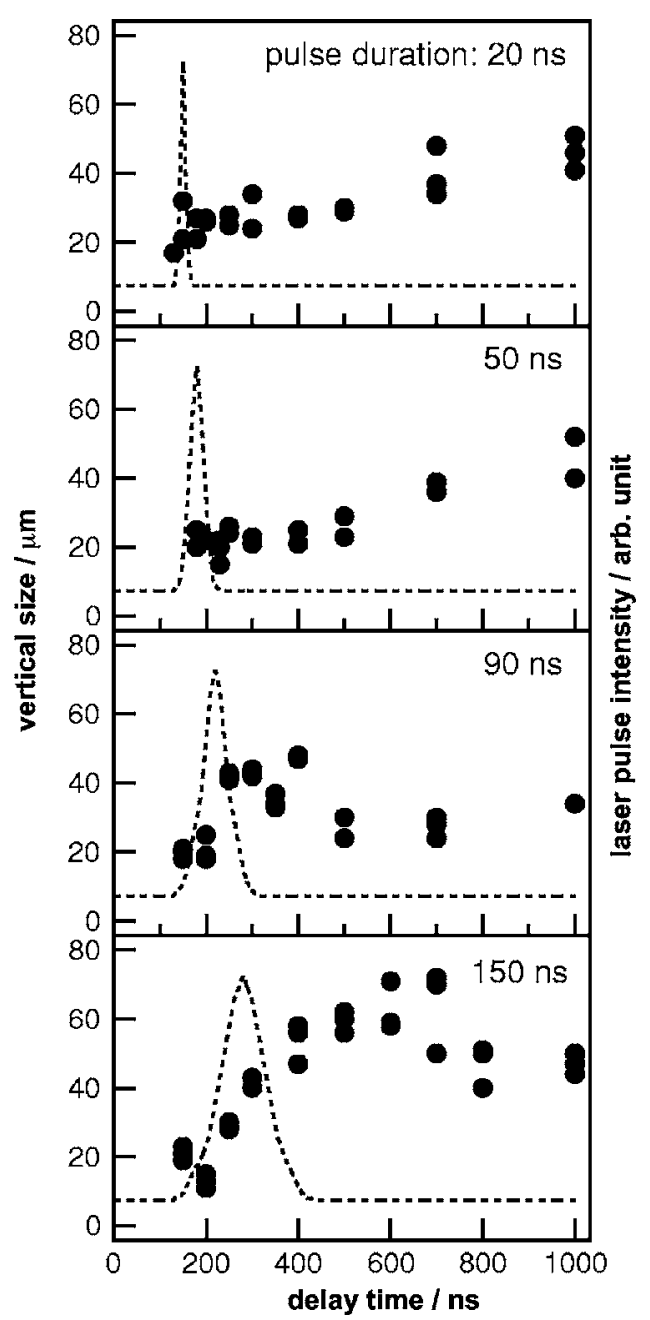

FIG. 7. The vertical size of the ablation plumes obtained by the irradiation of the 20-, 50-, 90-, and 150-ns pulses. Filled circles show the vertical size as a function of delay time, while the broken line represents the intensity profile of a laser pulse.

atoms. The gradual increase in pressure caused by such a procedure can lead to a drastic growth of the plume in the vertical direction. It seems that a slow expansion tends to give a hemispherical plume.

In the case of a shorter pulse, no marked increase in plume size in the normal direction is observed. The plume size is almost constant after the extinction of irradiation pulse intensity and the intensity of the emission is too low in this time range to obtain a clear spectrum. On the other hand, the plume size obtained for the 150-ns pulse is maintained at about $300 \mathrm{~ns}$ after the laser pulse extinction and the intensity in this time range is sufficiently high to give a clear spectrum. The long-lasting vertical size with a sufficiently high intensity for spectrum measurement is also observed in the result of the 90-ns pulse, but not in the results of the 20- and 50-ns pulses.

Finally, note that the earlier behavior clearly indicates the advantage of the use of a long nanosecond laser pulse for the spectrochemical analysis of a solid surface in liquid. When we carry out a laser ablation of a solid target immersed in liquid, the obtained ablation species are confined in the ablation plume by the surrounding liquid. The high 
density and high pressure of the plume due to the confinement effect result in the deformation of the profile of the obtained spectra. The present study has shown that the use of a long nanosecond laser attenuates this confinement effect and gives emission spectra similar to those obtained in a gas atmosphere.

\section{CONCLUSIONS}

To verify the effect of pulse duration on ablation plume formation, time-resolved images of ablation plumes obtained for various pulse durations have been investigated. Irregularly shaped images having some bright spots were observed particularly when a short pulse, such as a $20-n s$ pulse, was used. On the other hand, hemispherical plumes were observed for the irradiation with a 150 -ns pulse. From the images, the plume size in the lateral and vertical directions and emission intensity have been obtained. In the case of a shorter pulse, a remarkable confinement in the vertical direction and fast extinction were observed, which may be the cause of the deformation of the spectral profile. In the case of a longer pulse, on the other hand, the expansion in the vertical direction is evident and the light emission from the plume lasts longer. We conclude that further excitation by the later part of the longer pulse increases plume size, while keeping the atoms excited and suppressing the emission from the high-density spots, resulting in sharp atomic line spectra with a high emission intensity. The advantage of the use of a long nanosecond laser for the in situ spectrochemical analysis of a solid target in liquid is clear from the results presented.

\section{ACKNOWLEDGMENTS}

The authors thank Kokichi Hotta for his technical support. This work was financially supported by a Grant-in-Aid from the Japan Society for the Promotion of Science.

${ }^{1}$ F. Colao, R. Fantoni, V. Lazic, A. Paolini, F. Fabbri, G. G. Ori, L. Marinangeli, and A. Baliva, Planet. Space Sci. 52, 117 (2004).

${ }^{2}$ H. Balzer, M. Hoehne, V. Strum, and R. Noll, Spectrochim. Acta, Part B 60, 1172 (2005).

${ }^{3}$ B. Sallé, D. A. Cremers, S. Maurice, R. C. Wiens, and P. Fichet, Spectrochim. Acta, Part B 60, 805 (2005).

${ }^{4}$ V. Lazic, F. Colao, R. Fantoni, and V. Spizzicchino, Spectrochim. Acta, Part B 60, 1014 (2005).

${ }^{5}$ A. De Giacomo, M. Dell'Aglio, F. Colao, R. Fantoni, and V. Lazic, Appl. Surf. Sci. 247, 157 (2005).

${ }^{6}$ T. Sakka, T. Nakajima, and Y. H. Ogata, J. Appl. Phys. 92, 2296 (2002).

${ }^{7}$ T. Sakka, K. Takatani, Y. H. Ogata, and M. Mabuchi, J. Phys. D 35, 65 (2002).

${ }^{8}$ T. Sakka, S. Iwanaga, Y. H. Ogata, A. Matsunawa, and T. Takemoto, J. Chem. Phys. 112, 8645 (2000).

${ }^{9}$ T. Sakka, K. Saito, and Y. H. Ogata, J. Appl. Phys. 97, 014902 (2005).

${ }^{10}$ T. Sakka, K. Saito, and Y. H. Ogata, Appl. Surf. Sci. 197-198, 246 (2002).

${ }^{11}$ K. Saito, T. Sakka, and Y. H. Ogata, J. Appl. Phys. 94, 5530 (2003).

${ }^{12}$ T. Sakka, H. Oguchi, S. Masai, K. Hirata, Y. H. Ogata, M. Saeki, and H. Ohba, Appl. Phys. Lett. 88, 061120 (2006).

${ }^{13}$ H. Oguchi, T. Sakka, S. Masai, and Y. H. Ogata, Online Proceedings of LAMP2006, The Fourth International Congress on Laser Advanced Materials Processing, No. 06-134, 2006.

${ }^{14}$ T. Sakka, H. Oguchi, S. Masai, and Y. H. Ogata, Chem. Lett. 36, 508 (2007).

${ }^{15}$ See, for example, K. Saito, K. Takatani, T. Sakka, and Y. H. Ogata, Appl. Surf. Sci. 197-198, 56 (2002).

${ }^{16}$ A. E. Siegman, Lasers (University Science Books, Mill Valley, 1986), p. 1017. 